NEUROLOGICAL PICTURE

\title{
Monocular visual loss and platelet fibrin embolism to the retina
}
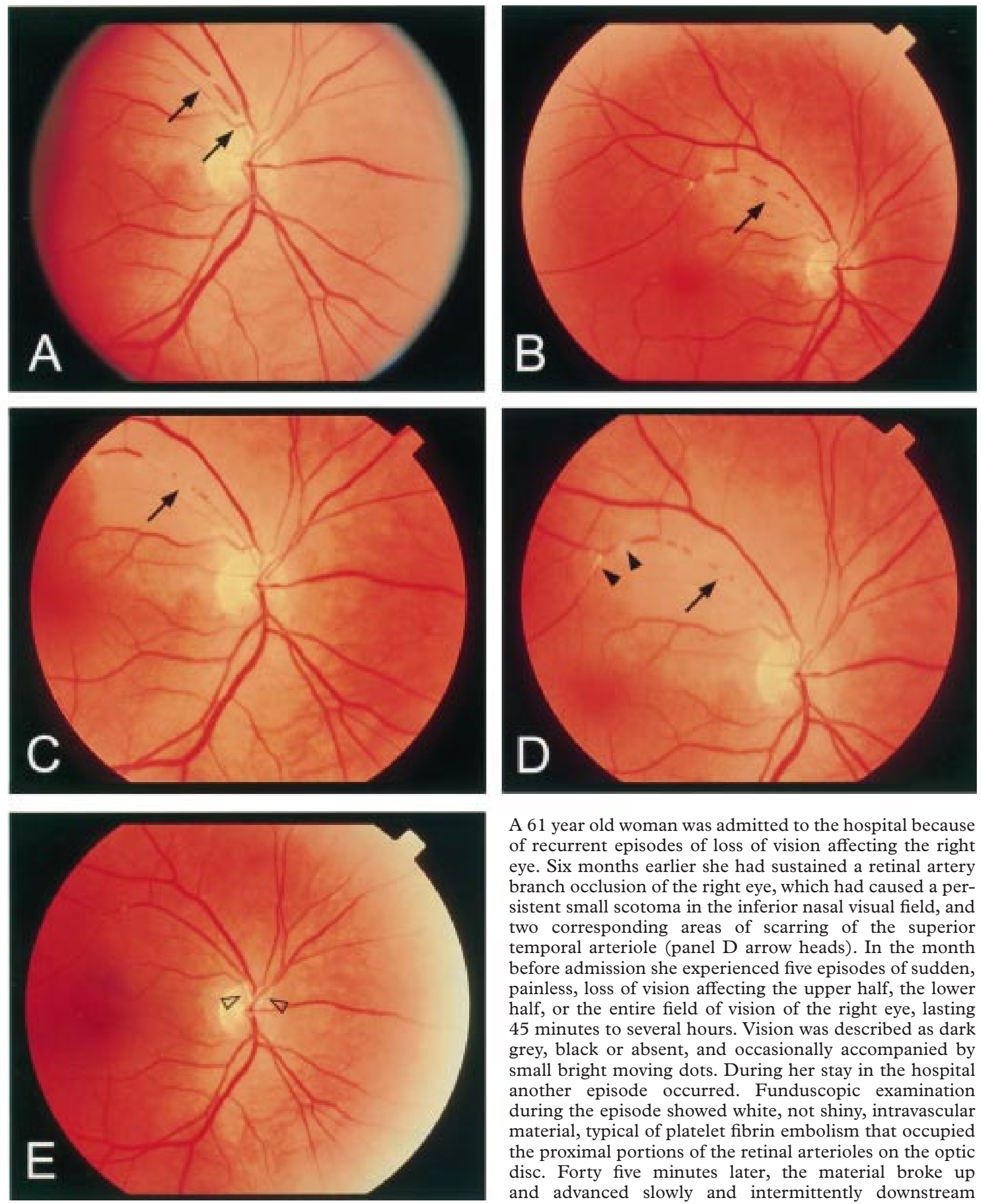

A 61 year old woman was admitted to the hospital because of recurrent episodes of loss of vision affecting the right eye. Six months earlier she had sustained a retinal artery branch occlusion of the right eye, which had caused a persistent small scotoma in the inferior nasal visual field, and two corresponding areas of scarring of the superior temporal arteriole (panel D arrow heads). In the month before admission she experienced five episodes of sudden, painless, loss of vision affecting the upper half, the lower half, or the entire field of vision of the right eye, lasting 45 minutes to several hours. Vision was described as dark grey, black or absent, and occasionally accompanied by small bright moving dots. During her stay in the hospital another episode occurred. Funduscopic examination during the episode showed white, not shiny, intravascular material, typical of platelet fibrin embolism that occupied the proximal portions of the retinal arterioles on the optic disc. Forty five minutes later, the material broke up and advanced slowly and intermittently downstream 
predominantly in the superior temporal arteriole (panels A, B, C, and D black arrows). Three hours later vision had returned to baseline, and the embolic material had resolved, but a new area of arteriolar sheathing or stationary platelet fibrin embolic material was seen in the proximal superior arterioles (panel E open arrow heads).

Platelet fibrin emboli were first observed by Miller Fisher during an episode of monocular visual loss in a patient with internal carotid artery disease. ${ }^{1}$ They are thought to originate from atherosclerotic arteries or from the heart, particularly from its valves, but have also been associated with coagulopathies, and sympathomimetic drug use. ${ }^{2}$ They typically appear as dull, grey-white, mobile intravascular material, occupying a long arteriolar segment before breaking up.

In the patient described here, an extensive investigation for a source of embolism including a transesophageal echocardiogram, and a contrast angiogram of the aortic arch, the carotid arteries, and the intracranial carotid system, including the ophthalmic artery, was unrevealing. She was discharged home and treated for 3 months with warfarin, followed by aspirin. She has remained asympto- matic up to the present time, 18 months later. Thus, as illustrated by this case, recurrent platelet fibrin embolism does occur in the absence of an evident embolic source. This raises the possibility of local formation of fibrin platelet embolic material due to an increased tendency for platelet aggregation.

CHRISTINE A C WIJMAN VIKEN L BABIKIAN Department of Neurology, Boston University School of Medicine, Boston, MA, USA; Boston Veterans Administration Medical Center, Boston, $M A$, USA

IPPOLIT C A MATJUCHA

Department of Ophthalmology, Boston University School of Medicine, Boston, MA, USA

Correspondence to: Dr C A C Wijman, Department of Neurology, University Hospital Utrecht, PO Box 85500, 3508 GA Utrecht, The Netherlands

1 Fisher CM. Observations of the fundus oculi in transient monocular blindness. Neurology 1959;9:333-47.

2 Miller NR Cerebrovascular disease. In: Miller NR, ed. Walsh and Hoyt's Miller NR. Cerebrovascular disease. In: Miller NR, ed. Walsh and Hoyt's
clinical neuro-ophthalmology. Baltimore: Williams and Wilkens, 1991:2210clinical

3 Magargal LE, Sanborn GE, Donoso LA, et al. Branch retinal artery occlusion after excessive use of nasal spray. Ann Ophthalmol 1985;17:500-1. 